\title{
Biodiversität und nachhaltige Landnutzung in Großschutzgebieten
}

\author{
Hubert Job ${ }^{1}$
}

Online publiziert: 3. November 2016

(c) Springer-Verlag Berlin Heidelberg 2016

Liebe Leserinnen und Leser,

aus internationaler Warte gibt es im Jahr 2016 drei gute Gründe, sich in dem vorliegenden Schwerpunktheft mit Großschutzgebieten zu beschäftigen:

Erstens wird der National Park Service, der die Großschutzgebiete in den USA betreut, 100 Jahre alt. Zählte man im ersten Erhebungsjahr 1904 für insgesamt sechs Nationalparke knapp 121.000 Besucher, waren es im Jahr 2015 über 307 Millionen, verteilt auf 372 Gebiete (NPS 2016).

Zweitens hat im März dieses Jahres im peruanischen Lima der vierte Weltkongress der Biosphärenreservate stattgefunden. Sein Thema lautete: „A New Vision for the Decade 2016-2025. UNESCO Biosphere Reserves for Sustainable Development“. Das Treffen von mehr als 1000 Delegierten endete mit der Anerkennung von 20 neuen Biosphärenreservaten auf allen Kontinenten. Damit sind nun insgesamt 669 UNESCO-Biosphärenreservate in 120 Ländern für die Umsetzung der globalen Nachhaltigkeitsagenda zuständig. Ebenso wurde der „Lima-Aktionsplan“ für das Weltnetz der Biosphärenreservate verabschiedet (UNESCO 2016).

Drittens wurde im September 2016 der World Conservation Congress der „International Union for Conservation of Nature and Natural Resources“ (IUCN) auf Honolulu, Hawaii (USA) durchgeführt. Die alle vier Jahre abgehaltenen Zusammenkünfte dienen den über 1300 Mitgliedern der Weltnaturschutzunion IUCN unter anderem dazu, die globale Naturschutzagenda als künftiges Arbeitsprogramm

Prof. Dr. Hubert Job

hubert.job@uni-wuerzburg.de

Lehrstuhl für Geographie und Regionalforschung, Julius-Maximilians-Universität Würzburg, Am Hubland, 97074 Würzburg, Deutschland für Nationalparke und andere Schutzgebiete im Hinblick auf die anstehenden weltweiten Herausforderungen, wie z. B. das Bevölkerungswachstum, den exzessiven Ressourcenverbrauch oder den Klimawandel, zu formulieren. Als übergeordnetes Ziel steht im Raum ,to improve how we manage our natural environment for human, social and economic development, [...] and work together to create good environmental governance, engaging all parts of society to share both the responsibilities and the benefits of conservation“ (IUCN 2016).

Was passiert diesbezüglich in Deutschland? Die Bundesregierung hat im Jahr 2007 die „Nationale Strategie zur biologischen Vielfalt“ (NBS) verabschiedet. Bis zum Jahr 2020 soll sich demnach ,die Natur auf $2 \%$ der Fläche wieder nach ihren eigenen Gesetzmäßigkeiten ungestört entwickeln und Wildnis entstehen" (BMU 2007: 28). Der derzeitige Anteil solcher Flächen wird auf maximal 0,6\% des bundesdeutschen Territoriums geschätzt (BfN 2016). Es gibt demnach noch genügend Luft nach oben. Der Strategie geht es um den Erhalt der Biodiversität und die Sicherung nachhaltiger Landnutzungssysteme, die damit verbundene Intensität der land- und forstwirtschaftlichen Nutzung sowie das Zulassen sekundärer Wildnis in unseren Kulturlandschaften. Die Variation an Biodiversität und das große Spektrum an Landnutzungssystemen finden sich in Deutschland idealtypisch in Großschutzgebieten.

Biodiversität ist in Deutschland nicht nur ein Naturschutzthema, sie spielt für eine nachhaltige Entwicklung eine elementare Rolle. Ihr Erhalt wirft Fragen auf, die originäre raumfunktionale Zuweisungen betreffen. Diese stellen sich überfachlich sowie überörtlich, zumeist auf regionaler Maßstabsebene. Großschutzgebiete haben Anhänger und Gegner, vor allem wegen der damit teilweise verbundenen allgemeinen Nutzungsverzichte und eventuell bestehender, besonderer Einschränkungen. In den vielfach kon- 
troversen Debatten um die Einrichtung oder Erweiterung von Großschutzgebieten geht es einerseits um Opportunitätskostenüberlegungen, andererseits um zu erwartende positive regionalwirtschaftliche Effekte. Damit sind Fragen angesprochen, die nicht nur aus naturschutzfachlicher Sicht interessant, sondern auch aus raumwissenschaftlicher Perspektive aktuell sind.

Seit der Wiedervereinigung nehmen in Deutschland Anzahl und Flächengrößen von Großschutzgebieten kontinuierlich zu. Großschutzgebiete sind landesrechtlich verankert, umfassen jeweils Areale von in der Regel über 10.000 ha und werden von einer eigenständigen Institution verwaltet. Sie umfassen mittlerweile etwa ein Drittel des deutschen Festlandterritoriums in drei unterschiedlichen Kategorien: Nationalpark, Naturpark sowie Biosphärenreservat. Der jüngste Nationalpark Deutschlands wurde an Pfingsten 2015 im Hunsrück offiziell eröffnet und das jüngste Biosphärenreservat, im Südschwarzwald gelegen, datiert vom 1. Februar 2016. Bayern ist gerade auf der Suche nach dem ,richtigen" Standort für den dritten Nationalpark im Freistaat. Grund genug, sich als Arbeitskreis der Akademie für Raumforschung und Landesplanung
(ARL) mit dem Thema zu beschäftigen und aus vor allem raumordnerischer Perspektive hierzu eine - naturgemäß lückenhafte - kritische Sachstandsanalyse vorzulegen. Die Ergebnisse dieser Analyse finden Sie in den Beiträgen dieses Schwerpunktheftes, verfasst von den Autoren des Arbeitskreises „Biodiversität und nachhaltige Landnutzung in Großschutzgebieten“.

\section{Literatur}

BfN - Bundesamt für Naturschutz (2016): Wildnisgebiete. https:// www.bfn.de/0311_wildnis.html (12.10.2016).

BMU - Bundesministerium für Umwelt, Naturschutz und Reaktorsicherheit (Hrsg.) (2007): Nationale Strategie zur biologischen Vielfalt. Berlin.

IUCN - International Union for Conservation of Nature and Natural Resources (2016): World Conservation Congress. http://www. iucn.org/secretariat/membership/about/union/members/worldconservation-congress (12.10.2016).

NPS - National Park Service (2016): National Park Service Visitor Use Statistics. https://irma.nps.gov/Stats (12.10.2016).

UNESCO - United Nations Educational, Scientific and Cultural Organization (2016): Man and the Biosphere Programme. http://www. unesco.org/new/en/natural-sciences/environment/ecological-sciences/ man-and-biosphere-programme (12.10.2016). 